\title{
A Narrative Approach to Detect the Vehicles using color, texture and edge based techniques
}

\author{
Ravi Gaurav ${ }^{1, a}$, Shubham Kumar ${ }^{1}$, Venkatesan $S^{2}$ and Ramesh Babu D.R. ${ }^{2}$ \\ ${ }^{1}$ Dept of CSE ,Dayanand Sagar College of Engineering, Bangalore,India \\ ${ }^{2}$ Professor, Dept of CSE ,Dayanand Sagar College of Engineering, Bangalore,India
}

\begin{abstract}
Vehicle recognition is the chief stride in observing the speeding vehicles in a thruway. The feature arrangements caught by a stationary camera demonstrate to us that there's a requirement for a vehicle location calculation which handles sudden light change furthermore the situations where the closer view converges away from plain sight. This paper gives us a study of different foundation subtraction systems that are utilized for recognizing the vehicles effectively. Vehicles proceeding onward street are of significance on the grounds that issues like movement blockage, monetary waste, sticking on the underpasses and over-extensions (if the vehicle going through is not of the passable size) are connected with them.Index Terms-Vehicle Detection, video sequences, foreground, background, MATLAB, RGB conversion.
\end{abstract}

\section{Introduction}

Hsu-Yung Cheng [1] utilized a pixel wise classification model for vehicle detection using Dynamic Bayesian Network (DBN). In numerous picture handling applications, closer view recognition is a vital step. Fitting recognition and ID of these frontal area protests just will prompt other handling like following. With a specific end goal to screen the vehicles in the expressway, we need to think seriously about the changing climate conditions and closer view protests that may converge away from plain sight later. The camera's perspectives will be unique in relation to distinctive positions of the camera which should likewise be considered in the recognition process. There is front perspective, back perspective, side view and top perspective. The stature at which the camera is put will likewise influence the identification's yield calculations. The camera details, the casing rate and range or separation secured by this altered camera likewise ought to be considered for proficient vehicle discovery. Also, as the camera will be altered and is inclined to part of aggravations to commotions, there will be clamor in the

\section{COLOR AND TEXTURE BASED VEHICLE DETECTION}

Shading and composition are two most critical qualities in picture preparing application. Visual shading difference is utilized to channel data present in every shading segment. (Tremeau et al. [2]) and to recognize among comparative dim scale intensities (Barilla-Perez \& Spann [2]). There have been both shading based and composition based features which ought to be uprooted for effective vehicle recognition. A few techniques have been proposed for vehicle identification. Vehicle identification should be possible utilizing foundation subtraction calculations and vehicle model based methods. Every calculation has its own focal points and detriments. Be that as it may, these routines attempt to recognize vehicles vigorously and productively. Utilizing any location calculation first the foundation and the closer view are isolated. Progressively vehicle identification, first the vehicles' shadow must be uprooted keeping in mind the end goal to stay away from errors in the recognition process. This paper gives a study on the current calculations that work on beating the issues said before. Area (II) gives a review on the strategy which utilizes both shading and surface for vehicle discovery. Segment (III) depicts a strategy that uses shading change. Extracting information from the image, calculating certain metrics based on this information and comparing the values of these metrics top predetermined values[10].

vehicle divisions. Be that as it may, reconciliation of these both will yield us better results in vehicle location. The street's shade is homogenous and they have the same surface all through. You can without much of a stretch identify vehicles making utilization of this. The vehicle's shade will demonstrate an enormous variety when contrasted and the street's shade. In any case, issue comes when the vehicle's shade is like the street's shade which can be understood by considering the composition. The initial phase in this system would be making a foundation

\footnotetext{
${ }^{a}$ Corresponding author: Ravi Gaurav, gaurav.ravi9@gmail.com
} 
model. A foundation model can be made utilizing recursive or non-recursive systems. Since clamor is included, you can utilize middle channel. The contrasts between the present casing and the foundation can be figured utilizing the elements of composition and the L* intensities part of $\mathrm{L}^{*} \mathrm{u}^{*} \mathrm{v}^{*}$ shading space. This system depends on a change location procedure that will consolidate both the power and surface contrasts between the present edge and the past edge. This strategies lives up to expectations useful for static foundations where there is no much distinction in the foundation's surface. Composition is viewed as a rich wellspring of data about the nature and three-dimensional state of items (Petrou (2006)). It comprises of complex visual examples and subpatterns with particular properties, for example, brilliance, shading and size (Materka \&Strzelecki(1998)). There exist diverse methodologies for composition demonstrating, for example, measurable models, auxiliary models what's more, models taking into account changes. Materka \& Strzelecki (1998) recommended that change systems allow the variety of spatial determination to speak to compositions at diverse scales. In this sense, Wavelet Transform is a feasible strategy to get time-recurrence limitation investigation and to recognize stationary and non-stationary compositions.

\subsection{Color And Edge Based Vehicle Detection}

Luo-wei Tsai, Jun-wei hsieh, Kuo-button fan et al.[3] proposed a methodology for identifying vehicles utilizing shading and edges. Dissimilar to the vast majority of the customary routines that uses the movement components to recognize vehicles, this technique presents another shading change model to locate the conceivable vehicle hopefuls. As the same vehicle can have distinctive hues in diverse climate and enlightenment conditions, vehicle identification taking into account hues weren't supported much. This proposed system, paying little respect to the fluctuating lighting conditions, is fit for distinguishing the vehicle pixels from the foundation. To begin with, every one of the info's shades pixel are anticipated on a shading space. At that point utilizing Bayesian system, vehicle hues can be recognized from the foundation. A vehicle will have distinctive size furthermore diverse introduction so a few vehicle theories are created from every pixel. The theories can be checked utilizing three critical components which are corners, edges and coefficients of wavelet changes. This system can be utilized to vigorously identify vehicles if there should arise an occurrence of static foundations.

\subsection{ALGORITHM}

A. Read/capture image
The picture is initially obtained from a live feature food or a current picture can be stacked from the memory. We shall consider that the obtained picture is in RGB group which is a genuine nature position for a picture [4], [5]. In MATLAB, thecaptured or imported RGB picture is three dimensional and every pixel is spoken to by a component of a network whosesize compares to the picture's measure.

\section{B. Converting RGB image to Black and White}

This procedure is done in two stages. The RGB picture is initially changed over to a two dimensional grayscale picture. The grayscale picture is only a lattice that holds the luminance (Y) estimations of the picture [6]. We get the luminance estimations of the picture by consolidating the RGB qualities utilizing the NTSC standard comparison (1) that duplicates the essential hues (red, green and blue) with coefficients in light of the affectability of our eyes to these hues as appeared: $Y=0.3$ $* R+0.59 * G+0.11 * B(1)$

The luminance picture is then changed over to high contrast (twofold) picture by a procedure called thresholding [7], [8]. A limit is set and the luminance of every pixel is contrasted and this edge. All values that are more noteworthy than this edge are supplanted with an intelligent one (white) and the qualities underneath this edge are supplanted by a legitimate zero (dark). The edge can be ascertained either by deciding the luminance estimations of pixels that relate to question areas in an example picture (i.e., by machine preparing) and afterward averaging these qualities using so as to bring about the limit, or a calculation that assesses the picture's histogram and amplifies the difference of power in the middle of items and foundations.

\section{Recognize edge of objects}

The picture is currently a two dimensional cluster with double components. Limits of the items are perceived by first setting a solitary pixel on the article foundation interface as a beginning stage and moving in a clockwise or counter clockwise course and scanning for other item pixels. The pixels may be looked either slantingly (in 8associated pixels) or edge-nearby pixels (in 4-joined pixels). By chasing for article pixels in a settled heading, the object's limit can be perceived [9].

\section{Colour recognition}

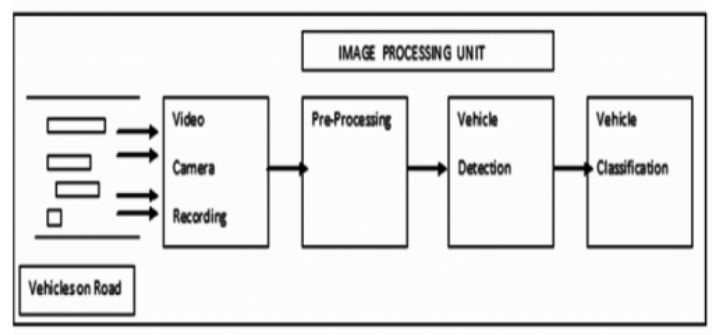

The items may be further assessed for their colour. This is finished by averaging the RGB content inside of the objects' limits. For instance an article with a generally 
high normal estimation of $\mathrm{R}$ over its surface, may have a shade of red. In MATLAB, the in pixel order can be utilized to gain the RGB data in a 1 X 3 grid over the whole protest surface which can be found the middle

\section{Fig1}

\section{E. Vehicle classification}

Isolation of Objects by Blob Analysis and Calculation of Convex Hull: The vehicle arrangement begins with separating every item and reshaping it into a close polygon shape with the goal that it mirrors the genuine vehicle's measurements from an aeronautical camera. The Convex Hull of every item is figured and the articles are reshaped into close polygon shape and more organized than they were before this stride.

\section{F: Post result of vehicle classification in MATLAB}

The work has been executed utilizing MATLAB rendition 7.6.0 (R2008a). There are initially 12 vehicles: 2 little, 4 medium and 6 major. In the wake of trimming, the top little auto is disposed of as an after effect of touching the picture's limit close to the medium auto on the right half of the street. Consequently, the aggregate number of remaining vehicles is 9: 1 little, 2 medium and 5 major vehicles which are appropriately distinguished and demonstrated utilizing red, green and blue rectangular boxes around them for little, medium and enormous vehicles separately as shown in the below figure:

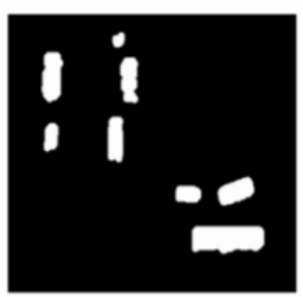

(a)

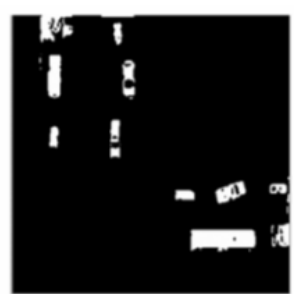

(b)

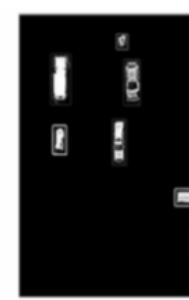

(c)
Fig2. : Output (a) Pre-processing; Algorithm; (c) Vehicle Classification

(b) Vehicle Detection

\section{G:PRE-PROCESSING TECHINIQUE:}

1.Histogram equalization: is a technique for adjusting image intensities to enhance contrast. Let $\mathrm{f}$ be a given image represented as a mr by mc matrix of integer pixel intensities ranging. from 0 to $\mathrm{L}-1$. $\mathrm{L}$ is the number of possible intensity values, often 256[17].

Histogram evening out is a procedure for modifying picture intensities to upgrade contrast. Let $\mathrm{f}$ be a given picture spoke to as a $\mathrm{mr}$ by $\mathrm{mc}$ grid of whole number pixel intensities running from 0 to $\mathrm{L}-1$. $\mathrm{L}$ is the quantity of conceivable power values, regularly 256 . Let $\mathrm{p}$ signify the standardized histogram of $\mathrm{f}$ with a container for every conceivable power. value of. Utilizing the standards of added substance blending, countless can henceforth be seen. fig1 shows Designing steps of the algorithm.

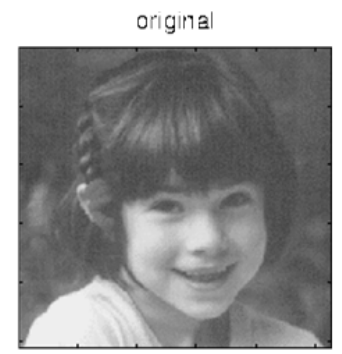

equalized image

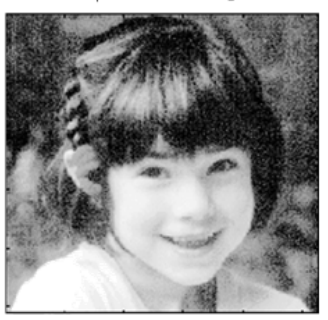

\section{Digital filtering:}

In picture preparing channels are for the most part used to stifle either the high frequencies in the picture, i.e. smoothing the picture, or the low frequencies, i.e. upgrading or identifying edges in the picture. A picture can be sifted either in the recurrence or in the spatial space. The main includes changing the picture into the recurrence space, increasing it with the recurrence channel capacity and re-changing the outcome into the spatial area. The channel capacity is formed to lessen a few frequencies and upgrade others. For instance, a straightforward lowpass capacity is 1 for frequencies littler than the cut-off recurrence and 0 for all others. The comparing procedure in the spatial space is to convolve the information picture $f(i, j)$ with the channel capacity $\mathrm{h}(\mathrm{i}, \mathrm{j})$. This can be composed as

$$
g(i, j)=h(i, j) \odot f(i, j)
$$

H: Input to the Pre-processing Phase: 


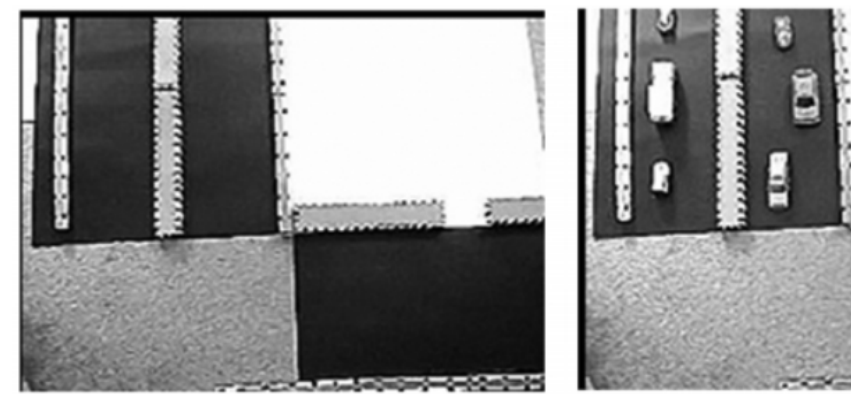

Fig: Reference and Current Image

I: Using Dual-Tree Complex Wavelet Transform (DTCWT)

As a structure, the Dual-Tree Complex Wavelet Transform (DTCWT) conquers a few challenges present in other Wavelet Transform techniques (Selesnick et al. (2005)) and offers the accompanying points of interest: - Nearly move invariance. At the point when the change is connected, the outcomes appear to be somewhat influenced by interpretations in the first info

- Directional selectivity. A subsequent arrangement of 6 diverse positive and negative bearings: $15^{\circ}, 45^{\circ}, 75^{\circ}$, $15^{\circ},-45^{\circ}$ and $-75^{\circ}$.

- Moderate repetition. The repetition presented by Complex Wavelets is 2d:1 for d Dimensions (Kingsbury (1999))

$\mathrm{K}$ : Application of vehicle detection

(a) Automated Car Wash Detection: A computerized auto wash framework needs to stay in a low-control utilization standby mode until a vehicle is recognized entering the auto wash.

Description: Previous innovation obliged the utilization of an inductive circle framework inserted into the ground. Introducing an inductive circle framework obliges cutting into an extensive segment of black-top or cement. After some time these inductive circles break and must be replaced. Using a Sure Cross M-GAGE remote sensor hub disposes of the requirement for a huge inductive circle. The gadget may be mounted over the ground or subterranean, obliging just a six to eight inch width opening. Vehicle vicinity is distinguished by a MGAGE Node that initiates the computerized auto wash framework to power up and triggers the section entryway.

(b)Indicating Traffic Levels in Vehicle Queues: Toll stalls on high-activity streets can rapidly go down amid top go times, bringing on cerebral pains for drivers and toll corner representatives. It is useful for specialists to know to what extent autos are holding up in every path so they can evaluate activity levels and distinguish moderate paths that may oblige additional consideration.

Description: Either M-Gage Vehicle Detection Sensors or R-Gage Radar Sensors can be utilized to screen an assigned checkpoint of every path and focus the length of time of time that every vehicle is ceased by then. L: Post Processing and Enhancement

In post processing stage we enhance the detection and per-forms the labelling of connected components to get vehicle objects. Size and aspect ratio constraints are applied again after morphological operations in the post processing to eliminate unwanted objects i.e., no vehicle objects. nonvehicle objects.

Here we utilize haar wavelet based future extraction [11] to upgrade the location on vehicles characterized by SVM. In all cases, characterization is performed utilizing GMM. Wavelets are basically a kind of multi determination capacity rough guess that take into account the progressive deterioration of a sign or picture. They have been connected effectively to different issues including article location [12] [13], face acknowledgment [14] and picture recovery [15]. Diverse reasons make the components separated utilizing Haar wavelets appealing for vehicle discovery. Some of them are they frame a conservative representation, they encode edge data which is an imperative element for vehicle recognition, they catch data from various determination levels furthermore there exist quick calculations for registering these elements [13] [12]. A few reasons make these elements appealing for vehicle identification. To begin with, they xxform a reduced representation. Second, they encode edge data, an imperative element for vehicle location. Third, they catch data from various determination levels. At last, there exist quick calculations, particularly on account of Haar wavelets, for processing these components.

\section{1: Flowchart showing the various step of processing of algorithm}

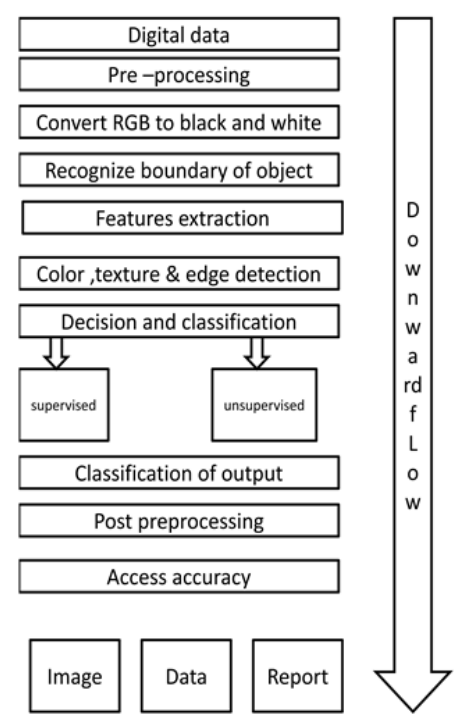




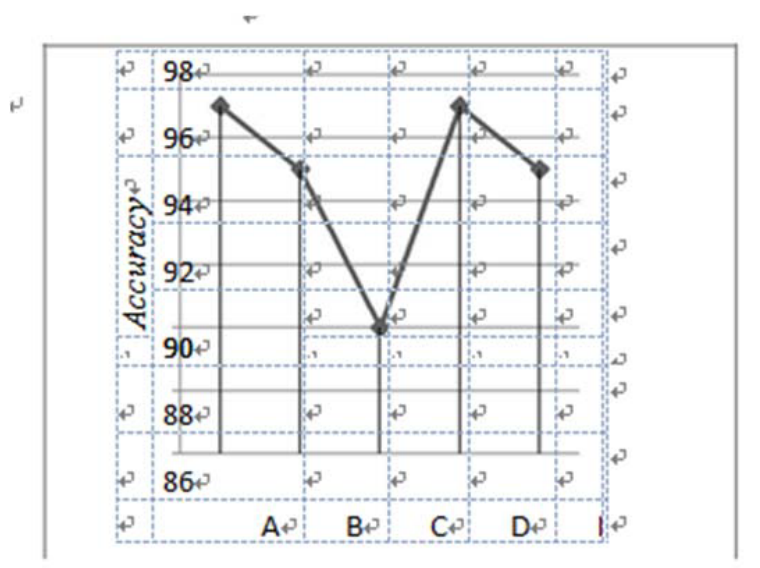

Methods $\leadsto$ Accuracy

Figure shown above diagrammatically representation of detection accuracy

Since method A and B is best among the all detection procedure.

Table -1

\begin{tabular}{|l|l|l|}
\hline $\begin{array}{l}\text { Name of vehicle detection } \\
\text { technique used }\end{array}$ & $\begin{array}{l}\text { ACCUR } \\
\text { ACY } \\
\text { RATE }\end{array}$ & False alarm \\
\hline $\begin{array}{l}\text { Color and texture based vehicle } \\
\text { detection (A) }\end{array}$ & $97 \%$ & Low \\
\hline $\begin{array}{l}\text { Color and edge based vehicle } \\
\text { detection (B) }\end{array}$ & $95 \%$ & Low \\
\hline Block matching algorithm (C) & $90 \%$ & High \\
\hline $\begin{array}{l}\text { Improved adative Gaussian } \\
\text { mixture model } \\
\text { (D) }\end{array}$ & $94 \%$ & High \\
\hline $\begin{array}{l}\text { Combining motion and detection } \\
\text { and background substraction (E) }\end{array}$ & $94 \%$ & High \\
\hline
\end{tabular}

\section{Conclusion}

This paper has given techniques on the various methods that available for vehicle detection in the day time with the image sequence obtained from a stationary CCTV camera at different views. Table 1 gives the accuracy in detection accuracy produced by applying each of the five methods. We have developed an algorithmic approach to vehicle detection. This not only reduces the complexity of the system but enhances its use in the areas which are too difficult to be detected by normal means. This algorithm can be applied on. The calculation talked about in this paper is a basic, yet viable system for examining the shapes and shades of articles. The idea of perceiving shapes on the premise of effectively possible. In our examination, the calculation was utilized to perceive the states of all cells and the Extent parameter of typical cancer cells was noted. As malignancy cells contrast from

\footnotetext{
${ }^{a}$ Corresponding author: Ravi Gaurav, gaurav.ravi9@gmail.com
}

solid cells fit as a fiddle and additionally shading, their Extent values and RGB substance don't coordinate those of cancer cells and can in this way be effortlessly distinguished and evaluated. The applications are not confined to the bio-restorative field but rather can reach out to any field that obliges order of distinctive articles in view of their physical appearance. This may incorporate quality assessment in sequential construction systems, counterfeit automated insight, PC vision, acknowledgment of vehicles at toll corners or movement signals for activity status determination, and so on.

\section{References}

1. Hsu-Yung Cheng, Chih-Chia Weng, and Yi-Ying Chen, - Vehicle Detection in Aerial Surveillance Using Dynamic Bayesian Networks\|, IEEE Trans. Image Process., vol. 21, no. 4, pp. 2152-2159, Apr. 2012.

2. Barilla-Perez, M. E. (2008). Colour-based Texture Image Segmentation, $\mathrm{PhD}$ thesis, University of Birmingham.

3. Koller D, Weber J. Huang T. Malik J. Ogasawara G. Rao B. Russell S.Towards robust automatic traffic scene analysis in real-time. inProceedings of the 33rd IEEE Conference on Decision and Control (Cat. No.94CH3460-3). IEEE. Part vol.4, 1994. 1994.

4. G. Wyszecki and W. S. Styles, "Color Science: Concepts and Methods, Quantitative Data and Formulae" (2nd edition New York: Wiley,1982).

5. R. S. Berns, "Principles of Color Technology" (3rd edition New York: Wiley, 2000).

6. J. L. Vincent, "Morphological Grayscale Reconstruction in Image Analysis: Applications and Efficient Algorithms", IEEE Transactions on Image Processing, vol. 2, pp. 176-201, 1993.

7. M. Sezgin and B. Sankur, "Survey over Image Thresholding Techniques and Quantitative Performance Evaluation", Journal of Electronic Imaging, vol. 13, no. 1, pp. 146-168, Jan. 2004.

8. N. R. Pal and D. Bhandari, "On Object Background Classification", International Journal Syst. Science, vol. 23, no. 11, pp. 1903-1920, Nov.1992.

9. F. Meyer, "Color image segmentation", Proceedings of 4th International Conference on Image Processing, pp. 523-548, 1992.

10. link.springer.com/chapter/10.1007\%2F978-3-64222456-0_4

11. Shalinee Patel, Pinal Trivedi, and Vrundali Gandhi, "2D Basic Shape Detection Using Region Properties", International Journal of Engineering Research \& Technology, vol. 2, no. 5, pp. 1147-1153, May 2013.

12. Zehang Sun, George Bebis and Ronald Miller, -Quantized Wavelet Features and Support Vector Machines for On-Road Vehicle Detection,\| Computer Vision Laboratory, Department of Computer Science, University of Nevada. 
13. C. Papageorgiou and T. Poggio, -A trainable system for object detection," International Journal of Computer Vision, vol. 38, no. 1, pp. 15-33, 2000.

14. H. Schneiderman, A statistical approach to $3 \mathrm{D}$ object detection applied to faces and cars. CMU-RI-TR-0006, 2000.

15. G. Garcia, G. Zikos, and G. Tziritas, -Wavelet packet analysis for face recognition," Image and Vision Computing, vol. 18, pp. 289-297, 2000.

16. C. Jacobs, A. Finkelstein and D. Salesin, -Fast multiresolution image querying," Proceedings of SIGGRAPH, pp. 277-286, 1995.

17. R. C. Gonzalez and R. E.Woods, Digital Image Processing, Third Edition, 2008. 